PRINT ISSN 1119-8362

Electronic ISSN 1119-8362
Full-text Available Online at

https://www.ajol.info/index.php/jasem

http://ww.bioline.org.br/ja
J. Appl. Sci. Environ. Manage.

Vol. 25 (5) 835 - 839 May 2021

\title{
Examination of the Seismic Characteristics of Onshore Reservoirs for Dom Gas Production Potentials in the Niger Delta Region of Nigeria
}

\author{
*INICHINBIA, S; HAMZA, H \\ *Department of Physics, University of Port Harcourt, Nigeria \\ Department of Geology, Ahmadu Bello University, Zaria, Nigeria \\ *Corresponding Author Email: sonny.inichinbia@yahoo.com, sonny.inichinbia@uniport.edu.ng
}

\begin{abstract}
The objective of this research work is to examine two prominent dom gas reservoirs H1000 and H4000 in onshore Niger Delta region of Nigeria for potential production activities using seismic wavelet and well-toseismic tie process to facilitate interpretation and evaluate dom gas (hydrocarbon) bearing formation. Well $\log$ and sidewall samples analyses show that the velocity attributes, high P-wave, S-wave and $V_{p} / V_{s}$ ratio characterize sediments with high concentration of dom gas. The wells validated the presence of dom gas with total gas in place on the field estimated at 4.017 trillion $\mathrm{ft}^{3}$, GIIP 5,228.683 bscf, CIIP 445.136 MMstb, and STOIIP 34.647 MMstb, URg 4,120.494 bscf and URc $219.208 \mathrm{MMstbl}, 90 \%$ of which resides in the two reservoirs. The hydrocarbon intervals are estimated at H1000 (8864 ft, TVDSS) and H4000 (9577 ft, TVDSS). The borehole information was combined with seismic data to confirm lateral continuity of the dom gas reservoirs.
\end{abstract}

\section{DOI: https://dx.doi.org/10.4314/jasem.v25i5.23}

Copyright: Copyright $\odot 2021$ Inichinbia and Hamza. This is an open access article distributed under the Creative Commons Attribution License (CCL), which permits unrestricted use, distribution, and reproduction in any medium, provided the original work is properly cited.

Dates: Received: 20 March 2021; Revised: 27 April 2021; Accepted: 07 May 2021

Keywords: Dom gas, horizons, synthetics, well-to-seismic tie, AVO, checkshot

This research work is a detailed seismic characterization of two prominent dom gas reservoirs H1000 and H4000 in onshore Niger Delta. The objectives of this work among others include the examination of the seismic characteristics of these reservoirs to help define the lateral extent of the dom gas away from the wells. The link between lithology and dom gas concentration by investigators from different parts of the world has been published by several authors and their investigations revealed that the formation of dom gas is strongly influenced by the lithology of the host sediments (Johnson and Max, 2006). Dom gas in our local parlance translates to what is known worldwide as gas hydrate or methane hydrate. Understanding of the total amount of dom gas in the world has some value in the deliberations of the possible role of dom gas in global climate change. The real issue for commercial development of the gas is whether it concentrated where it could be economically exploited! In this regard the global estimate becomes irrelevant, but it is useful for the exploration geophysicist to know how much dom gas prospects in trillion cubic feet are available and how they could be found. However, there is great hope for the exploration geophysicist as investigations conducted in basins worldwide reveal that dom gas bearing sands have the best potential for commercial development. This is because they have saturations usually exceeding $60 \%-80 \%$ of the pore space where petroleum system is optimized (Hardage and Roberts, 2006; Bellefleur et al., 2006). Early studies have shown researchers that dom gas could be the fuel of the future. They are considered the Earth's biggest potential source of hydrocarbon energy and could be a key element in meeting global natural gas demand.

\section{MATERIALS AND METHOD}

In this study, 20 x $17.5 \mathrm{~km}^{2}$ of anisotropic 3D PreSDM data of four seismic volume (full stack and three partial angle stacks - near, mid and far partial angle stacks) and five wells with suitable well data logs were used. Well-1 collected dom gas bearing sidewall samples, checkshot data and wireline logs. The sonic and density logs were edited, conditioned, calibrated and quality controlled. Spikes and odd values that may create false events were removed. The five (5) wells and the year of drilling is given as: well-1 (1961), well2 (1992) and well-3 (2006), well-4 (1974) and well-5 (1992). Petrel 2.0, Jason geosciences workbench, rokDoc, Techlog are some of the software packages used. The methods described by Johnson and Max (2006) and McGee (2006), were adopted for this work.

Location of the study area: The study area lies in the Niger Delta which is between latitudes $4^{\circ} \mathrm{N}$ and $6^{\circ} \mathrm{N}$ and longitudes $3^{\circ} \mathrm{E}$ and $9^{\circ} \mathrm{E}$, and is located $70 \mathrm{~km}$ 
northwest of Port Harcourt within licence OML 21 and OML 53 of the Niger Delta of southern Nigeria as shown in Figure 1.

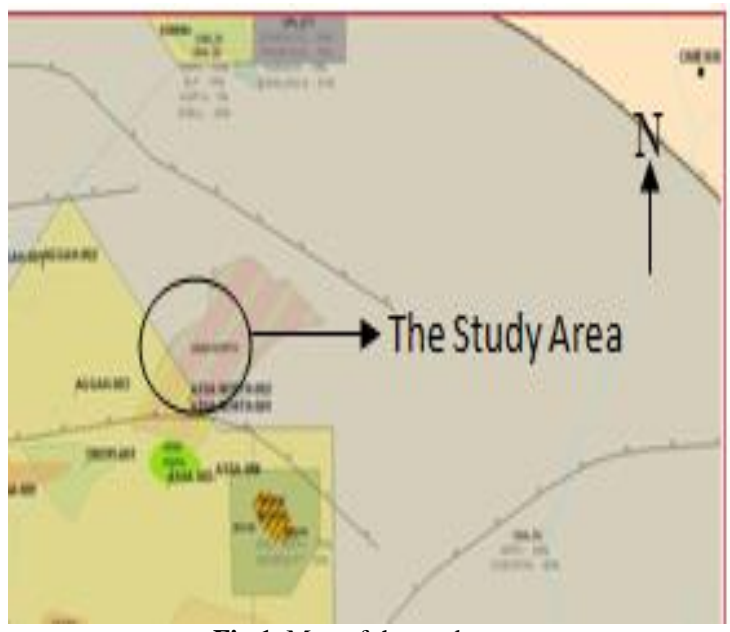

Fig 1. Map of the study area

\section{RESULTS AND DISCUSSION}

Figure 2 is the display of the well log panel of well-1 displaying some of the horizons, different types of logs, well markers, horizons, depths to the horizons, showing clear evidence of dom gas concentration through high resistivity, high elastic wave (P-and Swave) velocity, high attenuation, high $\mathrm{P}$ - and $\mathrm{S}$ impedance and low poisson's ratio (Hato et al., 2006). Six of the dom gas bearing sands are also highlighted. Seismic velocity is very sensitive to the presence of dom gas and so velocity could be used to characterize the formation, distribution and concentration of dom gas (Kumar et al., 2006). Figure 3 is the crossplots of the velocity attributes, $V_{p}, V_{s}$ and $V_{p} / V_{s}$ ratio while Figure 4 is the inverted $V_{p} / V_{s}$ ratio volume. Whereas the $V_{p}, V_{s}$ values increase, the $V_{p} / V_{s}$ ratio decreases. Since the measurement of $V_{p}, V_{s}$ and $V_{p} / V_{s}$ ratio as petrophysical properties help us to discriminate rock types and lithofacies, we apply this velocity attribute to the dom gas reservoirs to determine seismic based estimate of $V_{p}, V_{s}$ and $V_{p} / V_{s}$ in units that form dom gas systems. The seismic propagation medium is sand dominated and has a porosity of $21 \%$. Massive dom gas has $V_{p} / V_{s}$ ratio of $1.9-2.0$ (Hardage and Roberts, 2006). Decrease in the value of the $V_{p} / V_{s}$ ratio is indicative of increasing sediment rigidity with depth, as shown in Figure 3. The increased rigidity is caused partially by concentrations of dom gas that are uniformly distributed throughout the pore spaces of the reservoir units as disseminated clathrate that partly fill pore spaces and either bear a part of the overburden weight or float freely in the pore spaces or as alternating thin layers of dom gas free sediment and dom gas bearing sediment (Hardage and Roberts, 2006). Sidewall samples collected from well-1 defined how the dom gas is distributed throughout the host sediment. If gas migrating up a fault encounters appropriate conditions as well as sediments of sufficient permeability, dom gas could form within the pore spaces and act to cement the sediment grains, thus, increasing the shear modulus and bearing capacity of the sediments (Herron, 2006).

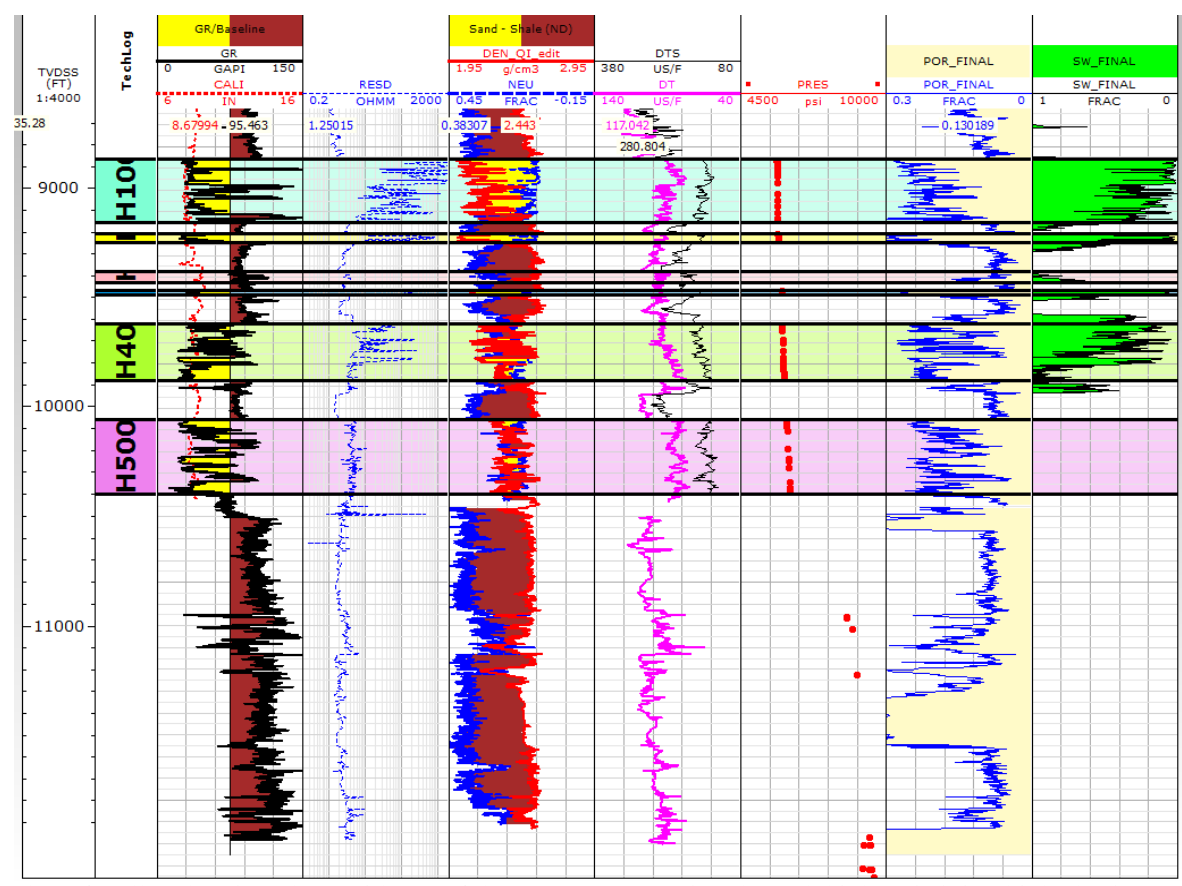

Fig 2. Composite log of well-1 showing clear evidence of dom gas concentration through high resistivity, high elastic wave velocity. Six of the dom gas bearing sands are highlighted.

INICHINBIA, S; HAMZA, $H$ 


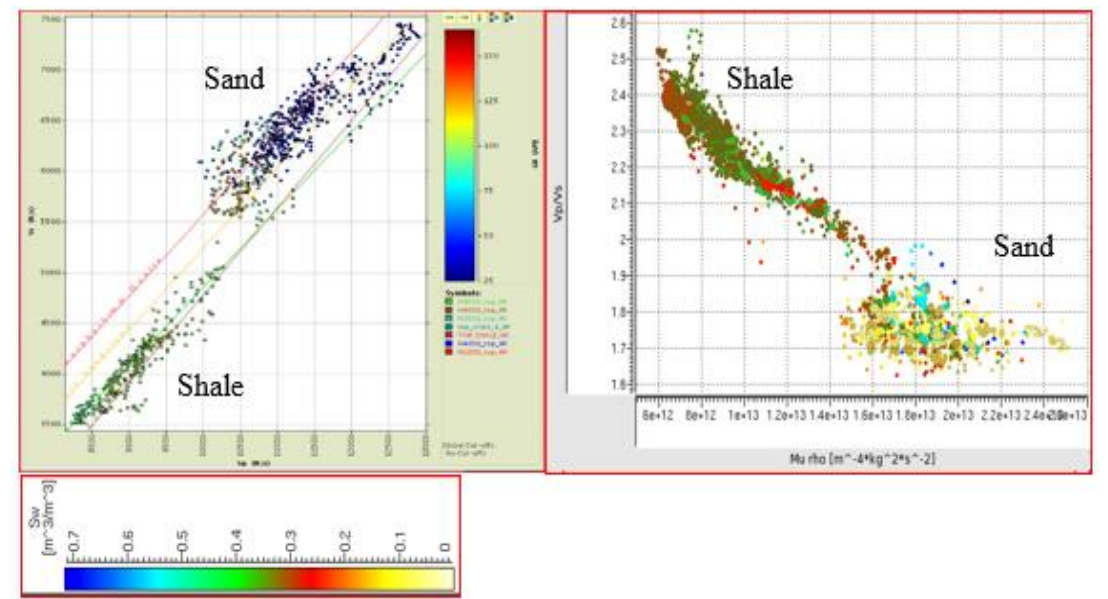

Fig 3. Crossplot of $V_{p}$ versus $V_{s}$ and $V_{p} / V_{s}$ with Mu rho colour coded to gamma ray and water saturation respectively relating to dom gas concentration for a sand dominated facies.

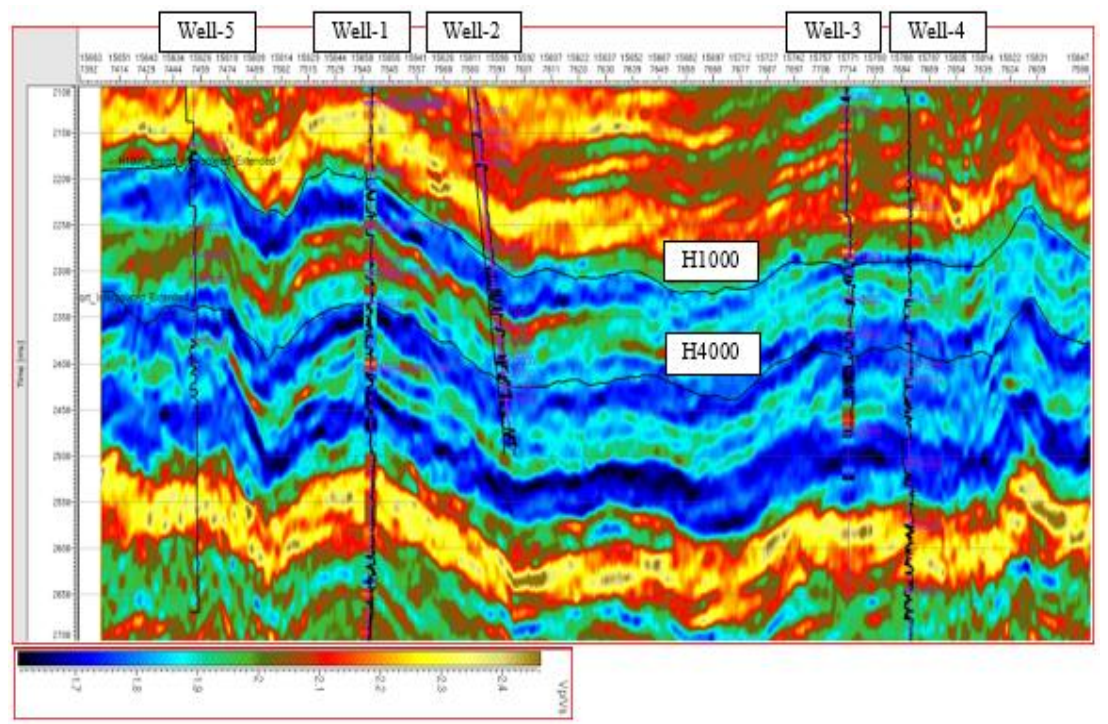

Fig 4. $V_{p} / V_{s}$ ratio volume with the five wells and some of their logs inserted with the horizons showing the extent of dom gas concentration in pore spaces.

Figure 5 is the synthetic modeling at well-1 using the anisotropic 3D seismic wavelet and the well-1 density and sonic logs. The logs were edited, condition and calibrated. Obvious data spikes were removed from the logs. Traces were extracted the section of the 3D data set through well-1. The time-to-depth conversion curve was derived from first arrival traveltimes using a zero offset checkshot data at well-1 as shown in Figure 6. Reflection coefficients were calculated from the calibrated density and sonic logs. Lateral continuity of the horizons was done with logs from the wells (Figure 5) and the result shows that the top, base and thickness of the reservoir zones A and B vary laterally when correlated across the wells. The calibrated sonic and density logs from well-1 were used to assess the well-to-seismic ties at the well locations. The strong correlation at the wells was fundamental to the evaluation of the spatial extent of the dom gas horizons around the wells from seismic data (Bellefleur et al., 2006). Synthetic traces from well-1 $\log$ s were compared to traces from the 3D seismic data set. For all cases a time-to-depth conversion curve was obtained from direct arrival traveltimes measured on a zero offset checkshot data acquired in well-1 and well-5, as shown in Figures 4 and 5 . The synthetic seismic traces were calculated by convolving the reflection coefficients obtained from the calibrated density and sonic logs with a wavelet extracted from the 3D and checkshot data sets using frequency matching. The data sets produced a wavelet that was near zero phase. Generally, the synthetic traces show negative amplitude troughs at the top and troughs at the base of the dom gas zones A and B. the traces also predict reflections (peaks and troughs) in the middle of the reservoir zones indicating changes in velocity corresponding to dom gas concentration 
within the interval. We also observed strong and continuous troughs at the base of the horizons. These reflections are excellent markers to determine the spatial extent of the dom gas zones around the wells. The 3D seismic and the synthetic traces show excellent correlation in time and depth in both reservoir zones and provided most of the details predicted on the synthetics. This resolution helped us to identify the variations within the horizons.

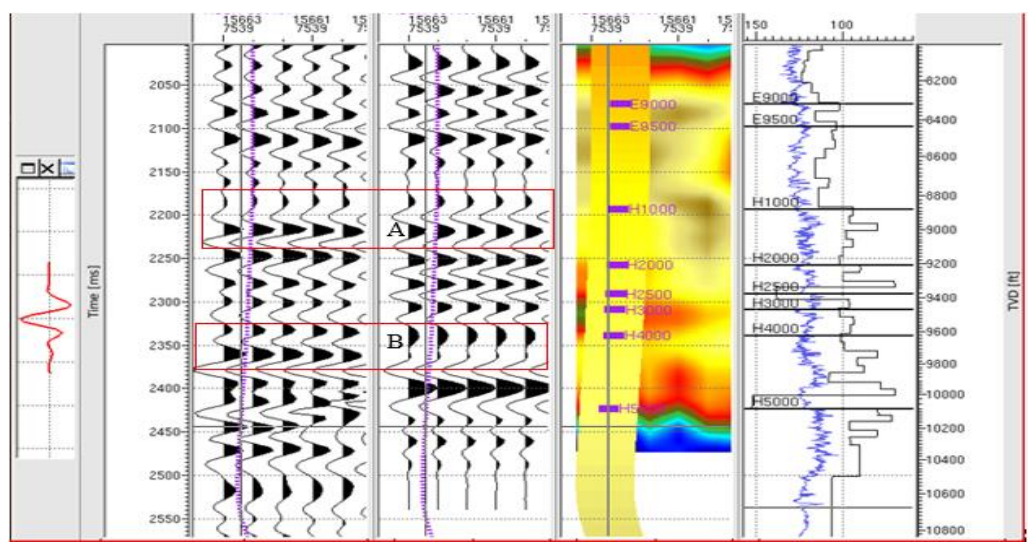

Fig 5. Synthetic modeling at well-1 using the anisotropic 3D seismic wavelet and the well-1 calibrated density and sonic logs.

The well-to-seismic tie is the first port of call to quality control (QC) the seismic data and to tie the data to meaningful geologic lithologies, reflectors and markers, thus, providing a basis for interpreting seismic events in geologic terms, establishing time-todepth relationship between the seismic data and well depths, estimate the wavelet and phase of the data, general quality control of the seismic and the well logs and understanding seismic resolution and tuning effects. Check shot data measured in a vertical well from a neighbouring field was borrowed to provide time-to-depth function to tie well logs in depth to the seismic in time. Accurate synthetic amplitudes were generated from the calibrated P-wave sonic log and density log. The calibrated sonic and density logs along with check shot data were used to create synthetics to calibrate the seismic (El-Behiry et al., 2020).

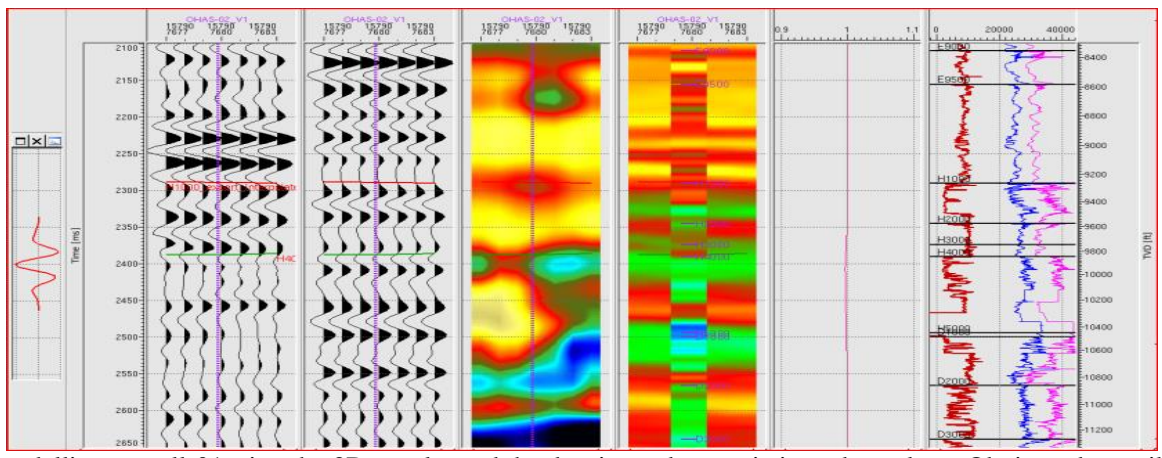

Fig 6. Synthetic modelling at well-01using the 3Dwavelet and the density and acoustic impedance logs. Obvioue data spikes were removed from the logs. Traces were extracted from the section of the 3D data set through well-01

Seismic amplitude is a function of acoustic impedance contrast at any acoustic interface. For this research work, amplitude variation with offset (AVO) seismic method was used because seismic attributes (amplitude and velocity) exhibit anomalous behaviour in dom gas bearing sediments; therefore, seismic attributes can characterize dom gas systems. Dom gas exhibits high seismic velocity and low density than surrounding sediments and the acoustic impedance of dom gas bearing sediments is generally higher than that of surrounding sediments. Occasionally, where high impedance (low porosity) sediments exist, the acoustic impedances of the dom gas bearing sediments and surrounding sediments are similar, causing smaller acoustic impedance contrast (lower seismic amplitude) at the interface of the dom gas bearing sediments. This decrease in amplitude is observed on seismic sections as amplitude blanking Low versus high saturation of hydrocarbons have long been a challenge when using seismic amplitude for predrill predictions, In order to look at the sensitivity of saturation we crossplotted the AVO attributes 
intercept and gradient as shown in Figure 7 and this showed that the time domain amplitude shows a good sensitivity to changes in saturation. This model is derived from properties given by a well log containing a relatively thick, porous sandstone layer and the saturations are based on Gassmann fluid substitution (Foster et al., 2020). From careful analysis, both reservoirs produce typical Class II AVO response.

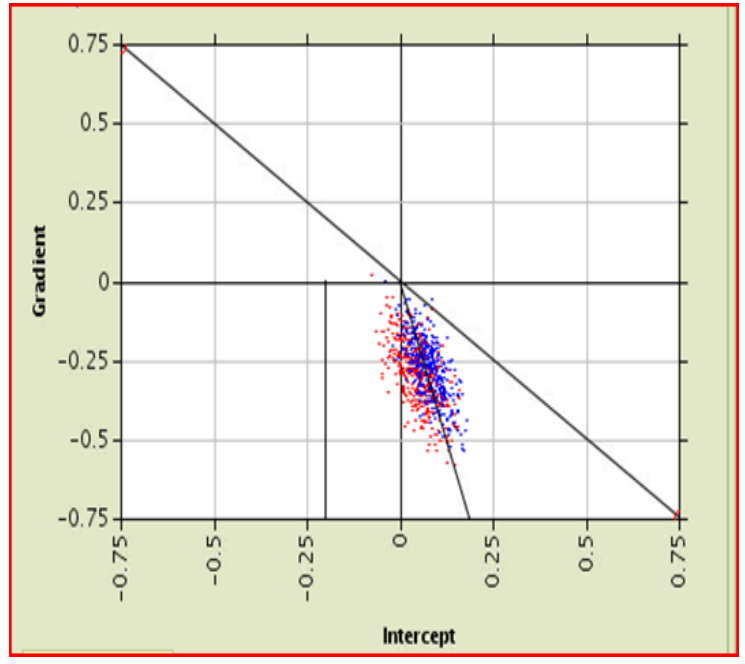

Fig 7. Time domain conventional crossplot between AVO gradient and AVO intercept showing high dom gas saturation level as a function of time windowed around the event of interest.

Concentration estimation: From the relation between dom gas fraction in sediments and observed seismic velocities, the $\mathrm{P}$-wave and $\mathrm{S}$-wave velocity anomalies (P- and S- waves velocities and impedances), with respect to a background velocity, we attempted the estimation of the dom gas distribution and concentration. An inversion scheme based on acoustic well $\log$ and density data from well-1 was adopted. For stable and quantitatively meaningful results, a wavelet that best represents the seismic data and excellent well-to-seismic correlation at the well site were a necessary prerequisite (Bellefleur et al., 2006; Dvorkin and Uden, 2006 and Kumar et al. 2006). The dom gas concentration estimated from drilling data compares well to that at the well location. This reserve's full field volume is estimated at GIIP/CIIP/STOIIP - 5,228.683 bscf/ 445.136 MMstb /34.647 MMstb and URg / URc - 4, 120.494 Bscf / 219.208 MMstbl with approximately $90.0 \%$ volume in two main reservoirs (i.e. H1000 and H4000). These reported values are only approximate. Sediments were found to contain nearly $3.0 \%-5.0 \%$ dom gas.

Conclusion: Dom gas was found to be concentrated in the coarse grained sands and totally absent from the shales and silts. Its production in Nigeria despite concerns about energy security has been a subject of local market conditions, technical capability and national focus. The same technology for safe conventional oil and gas operations in basins goes for the assessment of dom gas resources. The technology for dom gas development and production is drawing nearer.

Acknowledgements: The authors are thankful to Prahlad Basak, Dike Robinson, Francesca I. Osayande, Temitope Jegede, and the staff of the geosolutions and QI asset teams of the SPDC of Nigeria Limited. We also thank the SPDC of Nigeria Limited for providing the data.

\section{REFERENCES}

Bellefleur, G; Riedel, M; Brent, T (2006). Seismic characterization and continuity analysis of gas hydrate horizons near Mallik research wells, Mackenzie Delta, Canada. TLE, 25(5): 599 - 604

Dvorkin, J; Uden, R (2006). The challenge of scale in seismic mapping of hydrate and solutions. TLE, 25(5): $637-642$.

El-Behiry, MG; Al Araby, MS; Ragab, R.Z (2020). Impact of phase rotation on reservoir characterization and implementation of seismic well tie technique for calibration offshore Nile Delta, Egypt. TLE, 39(5): 346 - 352.

Foster, DJ; Zhao, Z; Kumar, D; Dralus, D; Sen, MK (2020). Frequency dependent AVO analysis. TLE. 39(5): $84-91$

Hardage, BA; Roberts, HH (2006). Evaluation of deepwater gas-hydrate systems. TLE, 25(5): 572 $-576$

Hato, M; Matsuoka, T; Inamori, T; Saeki, T (2006). Detection of methane-hydrate-bearing zones using seismic attributes analysis. TLE, 25(5): 607 $-609$

Herron, D (2006). Introduction to this special section: Gas hydrate. TLE, 25(5): 565

Johnson, AH; Max, MD (2006). The path to commercial hydrate gas production. TLE, 25(5): $648-651$

Kumar, D; Sen, MK; Bangs, NL (2006). Seismic characteristics of gas hydrates at Hydrate Ridge, offshore Oregon. TLE, 25(5): 610-614

McGee, TM (2006). A seafloor observatory to monitor gas hydrates in the Gulf of Mexico. TLE, 25(5): $644-647$ 